\title{
Study on the Contribution Rate of Shaanxi Rural Tourism to Rural Economy
}

\author{
Xiaoli Liu \\ Leisure Management school of Xi’an Eurasia University, Xi’an, Shaanxi, 710065
}

Keywords: Shaanxi rural tourism; rural economy; contribution rate

\begin{abstract}
Shaanxi rural tourism meets people's spiritual needs and psychological needs. It can promote the transformation from traditional agriculture to modern agriculture. It ultimately makes a contribution to promoting rural production development and increasing farmers' income, and promotes the development of agriculture and rural economy.
\end{abstract}

\section{Introduction}

Shaanxi Rural tourism is mainly based on the pastoral scenery, production and management activities, social and cultural customs, and other natural and cultural landscapes in rural areas. It continues to improve the infrastructure, attracting residents of closer distances to visit and consume, and realize tourism. The way consumers experience rural customs and return to nature. According to statistics, domestic Shaanxi Rural tourism received a total of 2.1 billion visits in 2016, of which employees reached 8.45 million, which benefited 6.7 million households. The revenue from Shaanxi Rural tourism exceeded 570 billion yuan. The huge economic benefits released by Shaanxi Rural tourism, its multiplier effect also constantly changes the face of rural areas, and plays an important role in rural economic development. It is of great significance to explore the impact mechanism and logic of Shaanxi Rural tourism on rural economic development.

\section{The Link between Rural Tourism and Rural Economy Development}

There is a close relationship between Shaanxi Rural tourism and rural economic development. In general, Shaanxi Rural tourism brings new economic growth points to rural development, while the development of rural economy provides complete infrastructure and quality services for Shaanxi Rural tourism. . Driven by the consumption of Shaanxi Rural tourism, the various resources of rural areas were further optimized and integrated, and the economic development of rural economy was promoted. Shaanxi Rural tourism is embedded in the rural economic development system, but it is a separate entity. The two are complementary to each other and have important implications for the overall development of society.

Judging from the result dimension, the development of Shaanxi Rural tourism in rural areas has the common goal of promoting the development of rural economy, that is, to promote the income of rural residents and to help rural residents get rid of poverty. In the process of achieving this goal, the two work together, that is, Shaanxi Rural tourism brings more consumption power to rural areas, broadens the income channels of farmers, and changes the way of rural economic development. The development of rural economy itself promoted the adjustment of the industrial structure of the industry, improved the infrastructure construction, updated the concept of the development of rural communities, and cultivated new farmers. Shaanxi Rural tourism itself as a concept and model of economic development has an important impact on the development of rural economy. Whether it is from the direct economic benefits it brings or indirect social changes, Shaanxi Rural tourism realizes the countercurrent of urban consumption power, that is, Consumption is not limited to cities, and the attractiveness of rural areas to cities has gradually narrowed the gap between urban and rural areas. The development of the rural economy spontaneously generates attraction for the city, and it exerts a certain influence in the development of the regional economy, creating a Shaanxi Rural tourism brand, which indirectly stimulates the development of Shaanxi Rural tourism.

While Shaanxi Rural tourism and rural economy can promote mutual promotion and mutual 
promotion, there is also a mutual restrictive relationship between the two. Shaanxi Rural tourism is limited by the actual situation of rural economic development in resource development and infrastructure supply. Due to the influence of Shaanxi Rural tourism brands and the impact of Shaanxi Rural tourism on the developing market space and potential market distance, the consumption power that Shaanxi Rural tourism can attract is limited, and the actual situation of rural economic development does not appear theoretically rural. The effect of tourism. Shaanxi Rural tourism can promote the development of the rural economy, but this development is only produced by factors such as market distance, tourism brands, consumption capacity, and the level of regional economic development. The ultimate effect of economic development in rural areas also determines whether it can provide sustainable driving force for Shaanxi Rural tourism.

\section{The Impact of Shaanxi Rural Tourism on Rural Economic Development}

The development mode of Shaanxi Rural tourism is different from the traditional agricultural development model of rural areas, and it simply shifts from agricultural farming to the supply of services. In the traditional mode of agricultural production, the rural labor force obtains more economic income. Affected by the economic dividends of cities and cities, the rural labor presents a situation of seasonal migration. It even leads to the problem of "hollow villages" in certain rural areas [1]. The development of Shaanxi Rural tourism creates a new economic source for the rural economy, which reduces the transfer problem of rural labor to some extent, effectively promotes the employment of farmers, increases the income of farmers, further improves the living standard of farmers, and can promote the new socialist countryside. Construction provides material security for the development of rural areas. At the same time, the development of Shaanxi Rural tourism has achieved a diversified pattern of rural economic development. The industrial chain formed by Shaanxi Rural tourism has promoted the development of the rural service industry and provided new economic growth points for rural economic development. The rural economy can combine agriculture and service industries, provide tourism villages with a good window and platform for the promotion of special agricultural products, expand the influence of rural development in rural areas, and further improve the level of rural economic development. In rural economic development, Shaanxi Rural tourism integrates various resources of rural villages, effectively promotes the adjustment of the agricultural production structure in the rural areas, and promotes the improvement of the overall industrial development through the interaction between industries and enhances the development capacity of the rural economy.

The development of Shaanxi Rural tourism has strengthened the link between rural areas and the outside world, and gradually eliminated the closed state of the village, which helps to promote the opening of farmers' thinking and improve the overall quality and ability. The development of tourism in villages has broadened the vision of farmers. Farmers are more exposed to the ideas and culture brought by outside consumers in the process of participating in Shaanxi Rural tourism service industry. Their development concepts and etiquette knowledge will change subtly and imperceptibly. The improvement of farmers' own quality and ability counteracts Shaanxi Rural tourism, and at the same time provides diversified talents for the development of rural economy and further promotes the sustainable development of rural economy. On the other hand, the development of Shaanxi Rural tourism helps to increase the importance and construction of infrastructure in rural areas. The precondition for the development of Shaanxi Rural tourism is to establish convenient transportation between rural areas and cities, so that urban tourism consumers can visit rural areas, and at the same time promote the development and improvement of rural network communication facilities. These infrastructure facilities Perfection laid the foundation for rural economic development.

Due to the fact that Shaanxi Rural tourism has a certain season, it has a certain negative impact on the income of Shaanxi Rural tourism. In order to reduce the impact of Shaanxi Rural tourism on the income of the busy season, we should give full play to the advantages of rural resources, develop diversified and refined products, thereby enhancing the income of Shaanxi Rural tourism, and promoting the development and progress of rural economy. In the process of developing 
diversified and refined rural products, the impact of seasons, the environment and other external factors should be eliminated, and the development trend of tourism and consumer fashion should be correctly grasped so as to establish a rich and diversified Shaanxi Rural tourism product system. Therefore, we have developed unique Shaanxi Rural tourism products and achieved sustained growth in Shaanxi Rural tourism revenues. Different types of Shaanxi Rural tourism should have different tourism products. They can classify tourism products into four types, and then develop pinpointed tourism products based on the characteristics of each type of tourism in the village. First, leisure and vacation travel products. These products are mainly based on the natural scenery of rural areas, creating a diversified, multi-grade leisure resort. The second is cultural tourism products. This type of product is based on rural hometowns of celebrities, folk customs, and rural culture such as water village cottages. It has a strong cultural atmosphere. Third, leisure and health tourism products. The main goal of the development of such products is to provide tourism and health care for the tourists, and to focus on tourism products such as mountain climbing, forest bathing, and fishing. Fourth, sports extreme tourism products to meet the needs of tourists outdoor sports. Therefore, Shaanxi Rural tourism should deeply study and analyze the needs of tourists, develop targeted, personalized, green, and humane tourism products according to the actual needs of the consumer market, and obtain the desired tourism while satisfying the needs of tourists. income.

In recent years, with the rapid development of society and economy, people have become more and more interested in Shaanxi Rural tourism. Every year, a large number of tourists go to the countryside to carry out various types of tourism projects. At the same time, local urban residents are the main source of Shaanxi Rural tourism. Followed by the surrounding cities. Therefore, in the process of development of Shaanxi Rural tourism, it is necessary to correctly view and evaluate its own advantages and disadvantages, strengthen the promotion and promotion of its own unique advantages, and innovate Shaanxi Rural tourism marketing strategies so as to attract more tourists. Nowadays, the rapid development of network technology provides convenient conditions for the marketing and promotion of Shaanxi Rural tourism, and can promote the promotion of Shaanxi Rural tourism through various forms of network platforms. At present, a large number of villages and towns travel and tourism have chosen to collaborate with travel agencies or surrounding influential areas, which is a marketing strategy adopted by village tourism. However, faced with the increasingly competitive countryside tourism market, all Shaanxi Rural tourism should adopt its own advantages to adopt innovative marketing strategies. For Shaanxi Rural tourism with higher economic strength, it is possible to develop a unique film and television marketing strategy by leveraging the advantages of the media, and transfer the characteristics of Shaanxi Rural tourism to various regions of the country or to the world in the form of publicity films, documentaries, and television advertisements. Various countries within the scope, so as to obtain good publicity. For Shaanxi Rural tourism with average economic strength, word-of-mouth marketing can be adopted, unique festival culture and other types of folk culture can be used to attract more tourists, and the integration of other marketing models, such as online marketing, is emphasized. In order to effectively enhance the popularity and appeal of Shaanxi Rural tourism. The development of Shaanxi Rural tourism has stimulated the establishment of rural leisure and cultural organizations. Many tourist families have established cultural performance teams in order to expand publicity, invigorate the cultural life in the village, and increase their appeal to tourists. The establishment of cultural and entertainment organizations has greatly improved the level of leisure among farmers, promoted the participation of villagers in recreational activities, and improved the quality of leisure and entertainment for villagers. The establishment of rural cultural and entertainment organizations, the increase of the diversity of public activities and the increase of villagers' leisure awareness have jointly promoted the enrichment of leisure activities for villagers.

\section{Conclusion}

From the four aspects of Shaanxi Rural tourism on farmers' labor lifestyle, consumption patterns, lifestyles, and cultural and entertainment lifestyles, we can see that Shaanxi Rural tourism has brought about great changes in farmers' lifestyles while promoting farmers' income. Make the 
peasants' lives more abundant and healthier.

\section{Acknowledgements}

Shaanxi Provincial Department of education, subject number (16JK2117),Subject name:Study on the contribution rate of Shaanxi Rural tourism to rural economy under the background of "tourism+"-An empirical analysis based on five samples in shaanxi.

\section{References}

[1] Luan An Jing. Shaanxi Rural tourism Rural Economic Status and Development Prospects [J]. Anhui Agricultural Sciences, 2007, (19)

[2] Zhao Chenghui, Chen Xiaoyue, Zhou Yanbo. Shaanxi Rural tourism Development Problems and Countermeasures [J]. Modern Agricultural Technology, 2009, (2)

[3] Dai Fumei. Shaanxi Rural tourism and Shaanxi Rural tourism Economic Development [J]. Technology Economy Market, 2007, (12)

[4] Zhang Huanzhou. An Empirical Study on the Impact of Shaanxi Rural tourism on Rural Economy: A Case Study of Xianhuashan Village, Pujiang County, Zhejiang Province [J]. Journal of Zhejiang Institute of Education, 2009, (3)

[5] Gaomouzhou. Research on the Relationship between Shaanxi Rural tourism Development and Rural Industrial Structure Adjustment [J]. Township Economy, 2008, (3) 